\title{
EFECTO DE LA FRECUENCIA DE RIEGO SOBRE LA EFICIENCIA DEL RIEGO Y LA PRODUCCIÓN DE UN CULTIVO DE FRESA
}

\author{
Gavilán, $P .{ }^{1}$, Ruiz, ${ }^{2}{ }^{2}$, Lozano, $D .^{3}$, Bohórquez, J.M. ${ }^{4}$, Molina, $F .^{5}$ \\ ${ }^{1}$ Investigador Titular. IFAPA Centro Alameda del Obispo, Apdo. 3092, 14080 Córdoba, \\ pedrod.gavilan@juntadeandalucia.es \\ ${ }^{2}$ Técnica Especialista Contratada. IFAPA Centro Alameda del Obispo. Apdo. 3092, 14080 \\ Córdoba, natividad.ruiz.baena@juntadeandalucia.es \\ ${ }^{3}$ Investigador contratado. IFAPA Centro Alameda del Obispo, Apdo. 3092, 14080 Córdoba, \\ david.lozano@juntadeandalucia.es \\ ${ }^{4}$ Técnico Especialista Contratado. IFAPA Centro Alameda del Obispo. Apdo. 3092, 14080 \\ Córdoba, juanm.bohorquez@juntadeandalucia.es \\ ${ }^{5}$ Director Departamento Agronómico. Gat Fertilíquidos, Niebla (Huelva). \\ pacomolina@gatfertiliquidos.com
}

\section{Resumen}

Este experimento ha permitido conocer los efectos del riego por pulsos de diferente duración y frecuencia sobre la eficiencia del riego y la producción de un cultivo de fresa. El trabajo se realizó sobre un suelo arenoso del entorno de Doñana. Se hizo un ensayo con cuatro tratamientos y cuatro repeticiones. Los tres primeros fueron regados diariamente con la misma cantidad de agua, aplicando pulsos de 5 (T1), 10 (T2) y 15 (T3) minutos de duración. El cuarto tratamiento (T4) recibió el riego de la parte comercial de la finca, con pulsos diarios de 15 minutos aproximadamente. En el tratamiento T3 se obtuvieron ahorros de agua del $44 \%$ sin pérdida de producción, con respecto a la parte comercial de la finca (T4), y pérdidas de cosecha de tan solo el $3 \%$ para el de pulsos de duración intermedia (10 minutos). El tratamiento de pulsos más cortos, T1, experimentó una pérdida de producción del $7 \%$, probablemente como consecuencia de la menor uniformidad de distribución del riego en estas condiciones y, por tanto, un mayor déficit hídrico en algunos puntos del invernadero. Por tanto, podemos concluir que los pulsos de riego entre 10 y 15 minutos serían los más aconsejables en este tipo de suelos. Además, pulsos muy cortos, como los del T1, deberían ser evitados. La eficiencia del riego del tratamiento más productivo (T3) fue del $79 \%$, mientras que la finca tuvo una eficiencia media del $42 \%$. Las producciones superaron los $1,000 \mathrm{~g}$ planta $^{-1}$ en el tratamiento de pulsos "largos" (T3) y en el T4. Además, las eficiencias en el uso del agua obtenidas en los diferentes tratamientos fueron muy similares, situándose alrededor de $23 \mathrm{Kg} \mathrm{m}^{-3}$.

\section{Summary}

The effect on crop yield of several daily drip-irrigation frequencies was investigated for strawberry (Fragaria $x$ anannasa) on sandy soil for one experiment conducted during 2013 to 2014 irrigation season. T1, T2 and T3 treatments were daily irrigated with 5, 10, and 15 minutes irrigation pulses and the same overall irrigation time. Therefore, these treatments received the same amount of irrigation water. T4 was daily irrigated according to the irrigation practices of the farmer. Irrigation frequency had little effect on yield. In all treatments production exceeded $900 \mathrm{~g} \mathrm{plant}^{-1}$, resulting in $3 \%$ and $7 \%$ reductions of significant yield of T2 and T1 treatments with respect to T3 and T4. No significant differences 
were detected between T3 and T4 treatments. Probably, the fall of T1 and T2 yields were due to a water stress induced by the distribution uniformity decrease when high irrigation frecuencies were applied. These results suggest that 15 minutes irrigation pulses are adequate for this type of soils. There was no yield benefit for short time irrigation pulses and, therefore, they were not advisable. T3 irrigation efficiency was $79 \%$ versus $42 \%$ for T4. In all irrigation treatments water use effciciency were around $23 \mathrm{Kg} \mathrm{m}^{-3}$.

\section{1- Introducción}

Es bien conocido que el riego localizado aumenta la eficiencia del riego al humedecer sólo la parte del suelo más cercana al sistema radicular (Bresler y col., 1982). Además, la frecuencia y descarga de los emisores determina el potencial de agua en el suelo y, por tanto, la distribución radicular y absorción de agua por las raíces (Coelho y Or, 1999). La frecuencia de riego óptima depende de factores tales como la textura del suelo, la extensión de sistema radicular, el frente de humedecimiento alrededor de las raíces, la evapotranspiración y el tipo de cultivo (Hanson y col., 2003). Los riegos frecuentes mantienen relativamente constante la humedad del suelo a lo largo del tiempo. Como desventaja, riegos diarios de muy alta frecuencia dan lugar a mayores tiempos de llenado de los laterales de riego y frecuente drenaje de los mismos después del corte del riego, lo que provoca problemas de baja uniformidad del riego como demostraron Lozano y col. (2014) en un experimento realizado sobre un cultivo de fresa en el entorno del P.N. de Doñana. Por el contrario, riegos poco frecuentes pueden causar estrés hídrico entre riegos en suelos de textura muy arenosa debido a la baja capacidad de almacenamiento de agua de estos suelos, lo que provoca una elevada percolación profunda por debajo de la zona explorada por las raíces. Desde la perspectiva del agricultor, un riego de menos frecuencia es más fácil de manejar, aunque este problema puede ser evitado con el uso de programadores de riego, y resulta más intuitivo.

Los laterales de riego por goteo usados en el cultivo de la fresa aplican entre 2.5 y 5 I $\mathrm{h}^{-1} \mathrm{~m}^{-1}$. Considerando un espaciamiento típico entre laterales de $1.1 \mathrm{~m}$, esto supone láminas de riego aplicadas que varían entre 2.3 y $4.5 \mathrm{~mm} \mathrm{~h}^{-1}$. Las necesidades de agua del cultivo en los meses de máxima demanda pueden alcanzan valores superiores a $5 \mathrm{~mm} \mathrm{~d}^{-1}$ (Gavilán y col. $^{a}, 2014$ ), que se deben cubrir, suponiendo una eficiencia del riego del $85 \%$, con riegos de hasta $6 \mathrm{~mm} \mathrm{~d}^{-1}$. El riego con cintas de $5 \mathrm{I} \mathrm{h}^{-1} \mathrm{~m}^{-1}$ supone, por tanto, tiempos de riego de hasta 80 minutos al día para cubrir las necesidades de agua del cultivo. Un riego de 160 minutos al día sería necesario si se usara una sola cinta de $2.5 \mathrm{I} \mathrm{h}^{-1} \mathrm{~m}^{-1}$. En la comarca del entorno del P.N. de Doñana son frecuentes los suelos arenosos, con un contenido de arena superior al $90 \%$ en algunos casos. Si el suelo explorado por las raíces no puede almacenar la mayoría del agua aplicada, parte de la misma se perderá por drenaje y la eficiencia del riego no será la óptima (Asolane y col., 2006). Por tanto, la aplicación de pulsos de riego de corta duración puede mejorar la eficiencia del riego (Batchelor y col., 1996).

Sin embargo, existe bastante incertidumbre sobre el efecto que tiene la duración y el número de pulsos de riego sobre la uniformidad de distribución (UD), la eficiencia del riego y la producción del cultivo de la fresa en la provincia de Huelva. Hanson y col. (2003) estudiaron el efecto de la frecuencia de riego sobre diferentes cultivos hortícolas (lechuga, pepino, cebolla y tomate de industria). Estudios similares fueron realizados previamente por Bucks y col. (1974), Freeman y col. (1976), Bucks y col. (1980), y Hutmacher y col., (1985). Por su parte, Linnemannstöns y col. (2013) realizaron un estudio similar sobre un cultivo de fresón. Durante la campaña de riegos 2013/2014, se realizó un estudio en condiciones de campo del efecto del uso de cintas de diferente caudal sobre la UD, cuando se aplicaban diferentes tiempos de riego, a escala de invernadero (Lozano y col., 2014) y sector de riego (Gavilán, 2014). El trabajo demostró que, a escala de sector de riego, tiempos de riego 
superiores a 10 minutos fueron necesarios para obtener valores de UD del $85 \%$, cuando se usaron cintas de $5 \mathrm{I} \mathrm{h}^{-1} \mathrm{~m}^{-1}$, las más frecuentes en la zona. Tiempos de riego de 5 minutos dieron lugar a UD del $79 \%$. Por tanto, en principio, un riego de 10 minutos sería la duración mínima del pulso que se debería aplicar para alcanzar una uniformidad calificada como "buena" cuando se utilizan cintas de $5 \mathrm{I} \mathrm{h}^{-1} \mathrm{~m}^{-1}$. Dando pulsos de riego de mayor duración (15 y 20 minutos) llegaron a obtenerse valores de UD del $93 \%$. Sin embargo, desconocemos el efecto que tiene la aplicación de pulsos de riego de diferente duración sobre la eficiencia de éste y la cosecha.

Los objetivos de este trabajo fueron: 1) la determinación de la duración óptima del pulso de riego cuando se cubren completamente las necesidades de agua del cultivo, es decir, en condiciones de riego no deficitario en relación con la eficiencia del riego y la producción 2) el cálculo de la productividad del agua de riego y de la eficiencia en el uso del agua para diferentes duraciones del pulso de riego.

\section{Material y métodos}

El ensayo se realizó en el T.M. de Almonte, cerca de la "Aldea de El Rocío", sobre una parcela comercial de fresón (Fragaria $x$ anannasa) de la variedad Sabrina propiedad de la empresa SUREXPORT S.L. El suelo de la zona del ensayo se clasifica como arenoso (clasificación USDA), con un $90 \%$ de arena y un 10\% de arcilla. La empresa realizó todas las labores de cultivo propias del cultivo (incluyendo el abonado). El manejo del riego fue responsabilidad del IFAPA. Además, la empresa se ocupó del manejo del riego necesario para construir los caballones, para mantener la estructura de los mismos antes del transplante y del riego por aspersión aplicado para asegurar el establecimiento de la planta tras el transplante. El transplante se realizó el 11 de octubre de 2013, con una densidad de plantación de 72,000 plantas ha-1 ${ }^{-1}$ aproximadamente. Los invernaderos fueron instalados el 11 de noviembre de 2013. La campaña finalizó el día 15 de mayo de 2014, cuando se dejó de recoger fruta de primera, destinándose a partir de ese momento toda la producción a industria.

A excepción del fertirriego, las plantas del ensayo recibieron las mismas labores que la parte comercial de la finca, no realizándose tareas de reposición de marras distintas a las realizadas por el agricultor. La recogida de la fruta se realizó de acuerdo con el calendario de recolección del resto de la finca, lo que no supuso ninguna ventaja para el ensayo en relación con el resto de la finca. Además, el cálculo de la producción por unidad de planta se realizó sin considerar el número de plantas muertas que se produjo a lo largo del experimento. Por tanto, los resultados del ensayo pueden ser perfectamente extrapolables al resto de la explotación, dado que podríamos calificarlo como un ensayo en condiciones de campo.

Se realizó un ensayo con cuatro tratamientos, con un diseño de bloques al azar con cuatro repeticiones. La unidad experimental fue un túnel completo de $70 \times 6.6 \mathrm{~m}^{2}$, por tanto, en el ensayo se compuso de 16 túneles, con una superficie total de $7,392 \mathrm{~m}^{2}$. Los tres primeros tratamientos estuvieron diseñados para cubrir completamente las necesidades de agua del cultivo, utilizando una eficiencia de riego del $85 \%$. Al primer tratamiento (T1) se aplicaron pulsos de 5 minutos, mientras que en el segundo y el tercero se dieron pulsos de 10 y 15 minutos, respectivamente. Por último, el tratamiento 4 correspondió al riego realizado en el resto de la finca, con pulsos de 15 minutos de duración aproximadamente, lo que permitió comparar las producciones con la producción comercial normalmente obtenida. El sistema de riego estaba compuesto por laterales de riego por goteo no autocompensante, con un espaciamiento entre emisores de $0.20 \mathrm{~m}$, capaces de aplicar $5 \mathrm{I} \mathrm{h}^{-1} \mathrm{~m}^{-1}$ a una presión de trabajo de 0.85 bar. Los datos de cosecha fueron analizados estadísticamente usando 
técnicas de análisis de la varianza. El test LSD fue usado para identificar tratamientos que fueran estadísticamente diferentes en producción.

La programación de riegos se realizó mediante el método de balance de agua en el suelo (Allen y col., 1998), utilizando la evapotranspiración de referencia estimada dentro de invernadero ( $\mathrm{ET}_{\mathrm{o}}$ inv) a partir del forzado del cultivo y valores del coeficiente de cultivo $\left(\mathrm{K}_{\mathrm{c}}\right)$ recomendados por Gavilán y col. (2014)a , calculados en función de la cobertura del cultivo, que alcanzó un valor máximo de 1.0 al final de la campaña, cuando el valor de cobertura máxima fue del $82 \%$. La $\mathrm{ET}_{\text {o inv }}$ se estimó usando un modelo basado en la radiación solar dentro de los túneles (Fernández y col., 2010), utilizando para ello el pronóstico meteorológico de la Agencia Estatal de Meteorología (AEMET). Diariamente, se comprobaban los valores del pronóstico con los estimados usando los datos meteorológicos medidos dentro de un invernadero que formaba parte del ensayo (Gavilán y col., 2014 ${ }^{\mathrm{b}}$ ). Para ello, se usó una estación meteorológica automática capaz de medir la temperatura y humedad relativa del aire (sonda HMP $45 \mathrm{C}$, Vaisala ${ }^{1}$ ) y la radiación solar (piranómetro CM3, Kipp and Zonen), controladas por un datologger CR10X (Campbell Scientific), perteneciente a la Red SIAR en Andalucía (Gavilán y col., 2008). Estos valores se muestran diariamente en Internet en la Web del IFAPA (www.juntadeandalucia.es/agriculturaypesca/ifapa/ria).

Para medir la evapotranspiración actual del cultivo $\left(E T_{c}\right)$ se instalaron en una repetición de cada uno de los tratamientos lisímetros de drenaje, realizados en poliéster reforzado con fibra de vidrio de $1.40 \mathrm{~m} \times 0.60 \mathrm{~m} \times 0.60 \mathrm{~m}$. La cantidad de agua aplicada a cada uno de los tratamientos fue medida utilizando contadores. Se instaló un contador en cabeza de cada tratamiento, lo que permitió conocer el volumen aplicado en los cuatro túneles que componían cada uno de ellos. Además, en los lomos donde se situaban los lisímetros, se instalaron contadores para medir la cantidad de agua aplicada a los mismos y, a partir de ese dato, calcular la cantidad que se suministraba al lisímetro. Más detalles sobre el experimento pueden consultarse en Gavilán y col. (2014 $\left.{ }^{\mathrm{a}}\right)$.

La humedad en el suelo se midió en cada uno de los tratamientos usando sondas capacitivas ECH2O (Decagon Devices ${ }^{1}$ ) y una sonda EasyAG (Sentek Technologies ${ }^{1}$ ) colocadas entre plantas. En ambos ensayos se monitorizaron en total 4 puntos de los diferentes tratamientos con las primeras, mientras que la segunda fue usada para monitorizar el contenido de humedad del tratamiento T1. La sonda EasyAG se conectó vía remota a un servidor de Internet, de manera que los valores de humedad del suelo pudieron ser visualizados de forma remota, lo que facilitó la toma de decisiones de riego. Los valores de humedad del suelo se utilizaron para corregir el almacenamiento semanal de humedad en el suelo al estimar los valores de $\mathrm{ET}_{\mathrm{c}}$, así como para conocer el contenido de humedad en cada uno de los tratamientos a lo largo del ciclo del cultivo. Esto permitió, además, ajustar el riego en función de las variaciones de humedad del suelo debidas a las condiciones meteorológicas que se produjeron a lo largo de la campaña.

\section{Resultados y discusión}

\subsection{Riego aplicado en cada uno de los tratamientos}

El calendario de riegos fue modificado a lo largo de la campaña en función de las condiciones meteorológicas y la humedad del suelo con el objetivo de aplicar el riego necesario para cubrir completamente las necesidades de agua del cultivo. La cantidad de

\footnotetext{
1 La información suministrada de marcas comerciales es meramente informativa y no supone la adhesión de los autores del trabajo a las mismas ni ningún indicador de su calidad.
} 
fertirriego aplicado fue de 3,982, 4,137 y 4,057 $\mathrm{m}^{3} \mathrm{ha}^{-1}$ en los tratamientos T1, T2 y T3, respectivamente (Tabla 1 ). Las diferencias de volúmenes de riego aplicados a estos tres primeros tratamientos fueron inferiores al $4 \%$, lo que puede considerarse adecuado en función de la incertidumbre de la medida realizada con los caudalímetros. El mes completo en que menos agua se aplicó fue noviembre $\left(311 \mathrm{~m}^{3} \mathrm{ha}^{-1}\right.$ al T2), mientras que en abril se alcanzaron los valores máximos de agua aplicada $\left(971 \mathrm{~m}^{3} \mathrm{ha}^{-1}\right.$ al T2).

Por su parte, la finca aplicó (T4) 7,301 $\mathrm{m}^{3} \mathrm{ha}^{-1}$, un $80 \%$ más de lo aplicado a la media de los tres primeros tratamientos. La diferencia entre el riego de la finca y el de los tres primeros tratamientos es consecuencia de los hábitos de riego de la finca y la falta de aplicación de un calendario de riegos basado en las condiciones meteorológicas y de cultivo. Por ejemplo, cuando se producen lluvias abundantes, los agricultores tienden a reducir el riego instintivamente, mientras que no lo hacen cuando hay días nublados y fríos sin precipitación, en los que la evapotranspiración también se ve reducida aunque no haya lluvias. Esto se puede apreciar si se comparan los valores de riego aplicado en esta misma finca durante las campañas de ensayos 2012/2013 y 2013/2014 en los meses de noviembre y marzo, meses con diferentes cantidades de lluvia en ambas campañas (las lluvias acumuladas caídas en estos dos meses fueron de 294 y $31 \mathrm{~mm}$ en la primera y segunda campaña, respectivamente). Los valores de $\mathrm{ET}_{0}$ fueron también superiores durante la campaña 2012/13, 429 frente a $398 \mathrm{~mm}$ de la campaña 2013/2014. Sin embargo, los tratamientos de riego óptimo no se vieron afectados por este hecho y la menor cantidad de riego aplicado fue consecuencia de una mejor gestión del mismo y no de las diferentes condiciones meteorológicas. Como se verá al analizar los datos de producción, esta última campaña ha sido calificada como "más seca", sin embargo se ha aplicado menos riego en los tratamientos de riego óptimo y las producciones han sido mayores que en la campaña anterior (datos no mostrados).

Tabla 1. Riego, evapotranspiración del cultivo $\left(E T_{c}\right)$, valor máximo del coeficiente de cultivo, $\left(\mathrm{K}_{\mathrm{c} \max }\right)$, eficiencia del riego, producción, productividad del agua y eficiencia del uso del agua en los diferentes tratamientos del ensayo.

\begin{tabular}{|c|c|c|c|c|c|c|c|}
\hline $\begin{array}{c}\text { Tratamient } \\
0\end{array}$ & $\begin{array}{c}\text { Riego } \\
\left(\mathrm{m}^{3} \mathrm{ha}^{-1}\right)\end{array}$ & $\begin{array}{c}E T_{c} \\
(\mathrm{~mm})\end{array}$ & $\begin{array}{c}\text { Eficiencia } \\
\text { del riego }\end{array}$ & $\mathbf{K}_{\mathrm{cmax}}$ & $\begin{array}{l}\text { Producción } \\
\left(\text { g planta }^{-1}\right)\end{array}$ & $\begin{array}{c}\text { Productivida } \\
\text { d del agua } \\
\left(\mathrm{kg} \mathrm{m}^{-3}\right)\end{array}$ & $\begin{array}{c}\text { Eficiencia } \\
\text { en el uso } \\
\text { del agua } \\
\left(\mathrm{Kg} \mathrm{m}^{-3}\right)\end{array}$ \\
\hline T1 & 3,982 & 293 & 0.74 & 1.0 & $943^{b}$ & 17.2 & 23.4 \\
\hline T2 & 4,137 & 301 & 0.73 & 1.0 & $986^{b}$ & 17.3 & 23.8 \\
\hline T3 & 4,057 & 319 & 0.79 & 1.0 & $1,016^{a}$ & 18.2 & 23,2 \\
\hline T4 & 7,301 & - & $0.42^{*}$ & - & $1,018^{a}$ & $10.1^{*}$ & $24.4^{*}$ \\
\hline
\end{tabular}

* Valores estimados usando un valor de $\mathrm{ET}_{\mathrm{c}}$ igual a la media de los tratamientos T1, T2 y T3.

\subsection{Evapotranspiración y coeficientes de cultivo}

Durante el ensayo, la evapotranspiración de referencia, suma de los valores obtenidos al aire libre $\left(E T_{0}\right.$ ) y bajo plástico ( $E T_{o}$ inv $)$, a lo largo de la campaña de riegos fue de $429 \mathrm{~mm}$. El valor de ET。 FAO-56 al aire libre fue de $518 \mathrm{~mm}$. La precipitación registrada en la estación meteorológica de Almonte, perteneciente a la Red de Información Agroclimática de Andalucía, fue durante este mismo periodo de $336 \mathrm{~mm}$. Esta última cifra se suministra como un indicador de la aridez de la campaña en que se realizó el experimento. La evapotranspiración de cultivo media medida con los lisímetros ( $E T_{c}$ ) fue de $304 \mathrm{~mm}$, variando desde $293 \mathrm{~mm}$ (T1) hasta $319 \mathrm{~mm}$ (T3). Sin embargo, estas diferencias no pueden ser calificadas como significativas dada la ausencia de medidas de lisimetría en todas las 
repeticiones. Las diferencias se debieron fundamentalmente a pequeñas variaciones en el crecimiento de las plantas dentro de los lisímetros. El mes de máxima evapotranspiración fue abril, en el que se alcanzó un valor acumulado de $91 \mathrm{~mm} \mathrm{mes}{ }^{-1}$, mientras que en noviembre el valor alcanzado fue de $7 \mathrm{~mm} \mathrm{mes}^{-1}$. La evapotranspiración de cultivo media a lo largo de la campaña fue de $1.5 \mathrm{~mm} \mathrm{~d}^{-1}$. La $E_{\mathrm{c}}$ estimada que se utilizó en la programación de los riegos fue de $271 \mathrm{~mm}$, inferior al valor medido en todos los tratamientos.

Para el cálculo del coeficiente de cultivo real se utilizó el cociente entre la $E T_{c}$, medida a través del balance de agua en los lisímetros de drenaje, y la $\mathrm{ET}_{\mathrm{o} \text { inv. }}$ El coeficiente de cultivo inicial si situó en torno a 0.1 , cuando la cobertura alcanzó valores del $10 \%$, aproximadamente a primeros de noviembre. Este coeficiente ascendió de manera progresiva hasta mediados de enero, alcanzándose valores cercanos a 0.6 , con coberturas cercanas al $50 \%$. A partir de ese momento creció más lentamente hasta alcanzar en marzo valores de 0.7 , con coberturas del $50 \%$. Finalmente, se produjo una nueva subida hasta alcanzar valores de $\mathrm{K}_{\mathrm{c}}$ iguales a 1 al final del ciclo, con una cobertura máxima superior al $82 \%$. Estos valores resultan bastante consistentes con los determinados durante la campaña 2012/2013 (Gavilán y col., 2014ª).

\subsection{Eficiencia del riego}

El conocimiento de la eficiencia del riego es un indicador de la cantidad de agua aprovechada por las plantas en relación con el total de agua aplicada mediante el riego. Además, indirectamente, es un indicador de la eficiencia del uso de los fertilizantes, pues el agua de drenaje lleva en nutrientes en disolución aportados por el fertirriego, provocando lo que se denomina contaminación difusa. Lo cierto es que existen muy pocos valores medidos de eficiencia del riego en los cultivos de fresa. Gavilán y col. (2014) en un experimento realizado en esta misma finca durante la campaña 2013/2014 midieron eficiencias de riego del 58, 67 y $83 \%$, cuando se aplicaron riegos de 7,957, 6,876 y $5,610 \mathrm{~m}^{3} \mathrm{ha}^{-1}$, respectivamente. Los valores que aparecen en la literatura de eficiencia en el riego localizado son meramente informativos, por tanto deben tomarse con cautela a la hora de manejar el riego y, en todos los casos, deben ser contrastados en la realidad. Así, un valor objetivo de eficiencia del riego del $85 \%$ resulta difícil de alcanzar sin un manejo adecuado del mismo, sobre todo en suelos muy arenosos, como los que existen en el entorno del Parque Nacional de Doñana.

En contra de lo esperado, el tratamiento que alcanzó la máxima eficiencia fue el T3 con un valor medio anual del $79 \%$, cercano al valor objetivo con el que se hizo la programación de riegos, que fue del $85 \%$. Este valor es comparable al citado por Fernández y col. (2012) para el cultivo de la judía en el ciclo de otoño de los invernaderos del Poniente de Almería (suministro relativo de agua -RIS- igual a 1.18) e incluso superior al del cultivo del pepino en esa misma zona (RIS igual a 1.62). La eficiencia del riego fue variable a lo largo de la campaña, partiendo de valores inferiores al $20 \%$ inmediatamente después de la plantación del cultivo, alcanzando valores en torno al $80 \%$ a partir del mes de febrero, y con valores máximos superiores al $95 \%$ a partir del mes de mayo. Los tratamientos T1 y T2 tuvieron eficiencias medias anuales del 74 y el $73 \%$, respectivamente. Finalmente, el valor estimado de eficiencia del riego del T4, correspondiente al riego de la finca, fue del $42 \%$.

Los datos anteriores pueden compararse con la campaña anterior si consideramos su finalización el día 15 de mayo de 2013. Se observa que el tratamiento de riego óptimo de la primera (T1) tuvo una eficiencia del $77 \%$, muy similar a las eficiencias obtenidas en los ensayos de riego óptimo de esta campaña (que variaron entre el 73 y el $79 \%$ ). Sin embargo, la eficiencia del riego aplicado en la finca (T4) durante la campaña 2012/2013 fue 
ligeramente superior (47\%) a la de esta última, como consecuencia del menor riego aplicado en la primera, como se ha explicado anteriormente.

En la Figura 1 podemos ver la diferencia entre un riego de $4,000 \mathrm{~m}^{3} / \mathrm{ha}$ (tratamientos T1, T2 y T3) y uno de aproximadamente $7,300 \mathrm{~m}^{3} /$ ha (tratamiento T4). Supone pasar de una eficiencia de hasta el $79 \%$ u otra del $42 \%$, e implica una reducción de los volúmenes de agua y fertilizantes en torno al $45 \%$. Además, en términos de impacto ambiental, la Figura 1 nos muestra los volúmenes de drenaje. Estos indican que en T4 la contaminación difusa es más del triple que la medida en T3. Por tanto, un riego de $7,300 \mathrm{~m}^{3} /$ ha contaminaría prácticamente tres veces más de lo que lo haría uno de $4,000 \mathrm{~m}^{3} / \mathrm{ha}$. Comparando estos resultados con los de la campaña pasada a fecha 15 de mayo, podemos ver que los tratamientos de riego óptimo de la campaña 2013/2014 produjeron un drenaje medio de 999 $\mathrm{m}^{3} / \mathrm{ha}$, del mismo orden del producido en la campaña anterior $\left(971 \mathrm{~m}^{3} / \mathrm{ha}\right)$. Por su parte, la finca aumentó el drenaje en un $21 \%$, pasando de 3,494 a $4,241 \mathrm{~m}^{3} / \mathrm{ha}$ de drenaje (Datos no presentados).

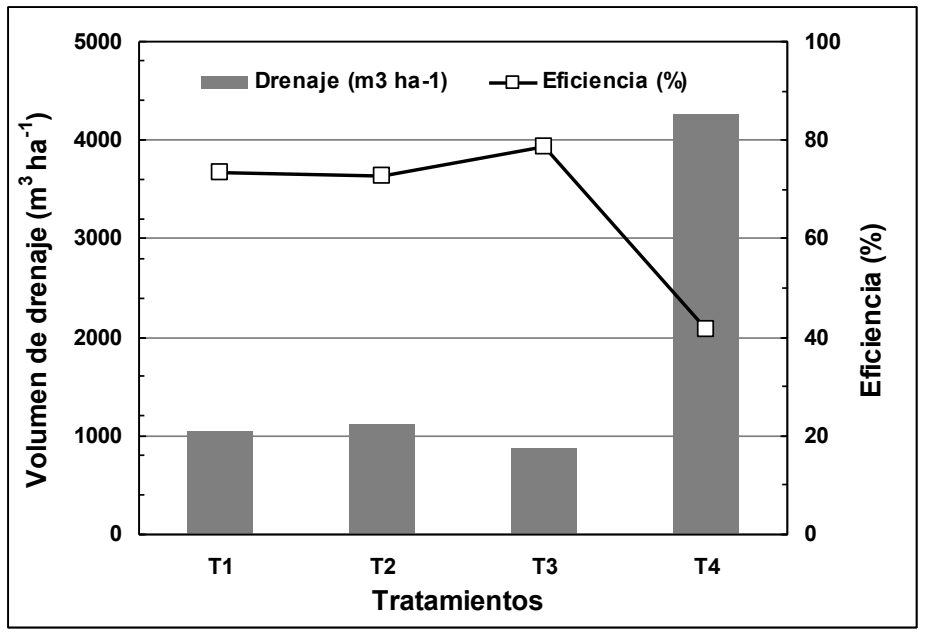

Figura 1. Volúmenes de drenaje $\left(\mathrm{m}^{3} \mathrm{ha}^{-1}\right)$ y eficiencia del riego (\%) en los diferentes tratamientos del ensayo de la finca Corraliza durante la campaña 2013/2014.

\subsection{Producciones, productividad del agua y eficiencia en el uso del agua}

En todos los tratamientos la producción de primera fue superior a $900 \mathrm{~g} \mathrm{planta}^{-1}$ (Figura 2). La máxima producción de los tratamientos con riego óptimo se obtuvo en el tratamiento de pulsos largos (T3), con un valor de 1,016 $\mathrm{g}_{\text {planta }}{ }^{-1}$ (equivalente a una producción de $7.3 \mathrm{~kg} \mathrm{~m}^{-2}$ ). Esta no fue significativamente diferente de la obtenida en el tratamiento que recibió un riego idéntico al del resto de la finca (T4), $1018 \mathrm{~g}_{\text {planta }}{ }^{-1}$, a pesar de que este tratamiento aplicó un $80 \%$ más de agua que el T3 (Tabla 1). Los tratamientos de pulsos más cortos, T1 y T2, tuvieron producciones de 943 y $986 \mathrm{~g}$ planta $^{-1}$, no existiendo diferencias significativas entre ellos. Sin embargo, las diferencias de estos dos últimos tratamientos fueron significativamente diferentes de T3 y T4. A pesar de ello, el ahorro de agua y fertilizantes de T1 y T2 respecto a T4, un 45 y un $43 \%$, respectivamente, podría justificar la merma de producción, que viene siempre a producirse a partir del mes de abril, cuando los precios de la fruta han disminuido considerablemente (datos no presentados). De cualquier forma, es importante destacar, por tanto, que se apreciaron pocas diferencias entre los tratamientos que recibieron cantidades iguales de agua con pulsos de diferente duración, al contrario de lo que podría pensarse a priori. Incluso, el tratamiento de pulsos 
"largos" fue significativamente más productivo, alcanzando al valor de referencia de la finca (T4). Es posible que bajo condiciones de riego óptimo, el incremento de la uniformidad de distribución que se produce al aplicar pulsos más largos compense la pérdida de eficiencia que se produce como consecuencia de una mayor percolación profunda.

La productividad del agua de riego en cada uno de los tratamientos fue de 17.2, 17.3, 18.2, $10.1 \mathrm{~kg} \mathrm{~m}^{-3}$, para los tratamientos T1, T2, T3 y T4, respectivamente. La productividad del agua de los tratamientos de riego óptimo (T1, T2 y T3) fue similar a la del riego del cultivo pimiento y de la judía en los invernaderos sin climatizar del Poniente de Almería (Fernández y col., 2012). Finalmente, las eficiencias en el uso del agua, calculadas como la relación entre la producción y la $\mathrm{ET}_{\mathrm{c}}$, obtenidas en los cuatro tratamientos fueron muy similares, oscilando entre 23 y $24 \mathrm{~kg} \mathrm{~m}^{-3}$.

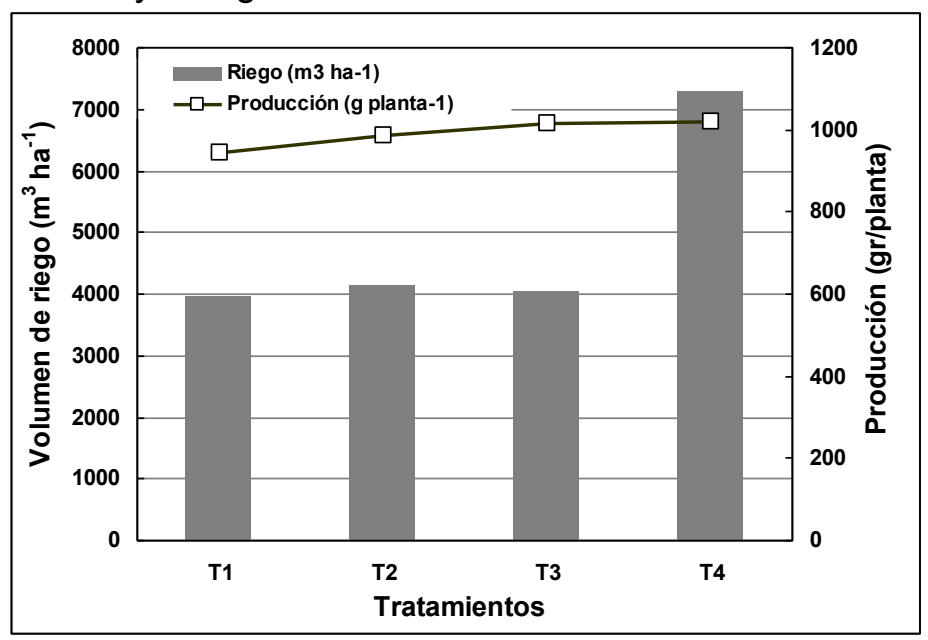

Figura 2. Volumen de riego aplicado $\left(\mathrm{m}^{3} \mathrm{ha}^{-1}\right)$ y producción (g planta $\left.{ }^{-1}\right)$ en cada tratamiento del ensayo de la finca Corraliza durante la campaña 2013/2014.

\section{Conclusiones y recomendaciones}

De los resultados anteriores se pueden extraer varias conclusiones. En primer lugar, la utilización de un calendario de riego basado en datos meteorológicos, coeficientes de cultivo y control de la humedad del suelo se han mostrado herramientas muy útiles a la hora de obtener ahorros significativos de agua sin pérdida de producción respecto a la parte comercial de la finca. Así se han llegado a obtener ahorros de agua del $44 \%$ sin pérdida de producción en el tratamiento de pulsos largos y pérdidas del $3 \%$ para el de pulsos intermedios (10 minutos de duración del pulso de riego), con ahorros de agua del $43 \%$. El tratamiento de pulsos más cortos, T1, experimentó una mayor pérdida de producción (alrededor del 7\%), probablemente como consecuencia de la caída de la UD del riego en estas condiciones y, por tanto, al aumento de déficit de riego en algunos puntos del campo. Por tanto, podemos concluir que pulsos de riego de 15 minutos serían los más aconsejables en este tipo de suelos. Además, pulsos muy cortos de riego, como los del T1, deberían ser evitados. El coeficiente de cultivo medido estuvo fuertemente influenciado por la cobertura del cultivo. El $\mathrm{K}_{\mathrm{c}}$ máximo alcanzó valores de 1 , con coberturas del $82 \%$. La eficiencia del riego del tratamiento con riego óptimo más productivo (T3) fue del $79 \%$, mientras que la finca tuvo una eficiencia del $42 \%$. Esto último indica que un $58 \%$ del agua aplicada no fue utilizada por el cultivo. Todos los tratamientos tuvieron producciones superiores a $900 \mathrm{~g}$ planta ${ }^{-1}$. Las producciones superaron los $1,000 \mathrm{~g} \mathrm{planta}^{-1}$ en el tratamiento de pulsos "largos" (T3) y en el regado como la parte comercial de la finca. Las diferencias entre los tratamientos que recibieron cantidades iguales de agua con pulsos de diferente duración 
fueron relativamente pequeñas, como podría pensarse a priori. Incluso, el tratamiento de pulsos "largos" fue igual de productivo que el correspondiente al riego de la finca y más productivo que los tratamientos T1 y T2. Además, las eficiencias de riego y las eficiencias en el uso del agua obtenidas fueron muy similares en los tratamientos T1, T2 y T3. Es posible que bajo condiciones de riego óptimo, el incremento de la uniformidad de distribución que se produce al aplicar pulsos más largos compense la pérdida de eficiencia que se produce como consecuencia de una mayor percolación profunda.

\section{Agradecimientos}

Este trabajo ha sido financiado por el proyecto CONECTA-SAR (PP.TRA.TRA2013), cofinanciado con fondos FEDER. Además, ha sido cofinanciado por la empresa SUREXPORT S.L. Agradecemos a Andrés Morales y a los técnicos de esta empresa María José, Mari Luz y Juan su colaboración en este trabajo.

\section{Bibliografía}

Allen, R.G., Pereira, L.S., Raes, D., Smith, M., (1998) Crop evapotranspiration. Guidelines for computing crop water requirements. FAO irrigation and drainage paper 56. FAO, Roma.

Assouline, S., Möller, M., Cohen, S., Ben-Hur, M., Grava, A., Narkis, K., Silber, A., 2006. Soil-Plant System Response to Pulsed Drip Irrigation and Salinity: Bell Pepper Case Study. Soil Science Society of American Journal, 70:1556-1568.

Batchelor, C., Lovell, C., Murata, M., 1996. Simple microirrigation techniques for improving irrigation efficiency of vegetable gardens. Agricultural Water Management. 32:37-48.

Bresler, E., McNeal, B.L., Carter, D.L., 1982. Saline and sodic soils. Advances Series in Agricultural Sciences. Vol. 10. Springer-Verlag. Berlin.

Bucks, D.A., Erie, L.J., French, O.R., 1974. Quantity and frequency of trickle and furrow irrigation for efficient cabbage production. Agronomy Journal, 66(1):53-57.

Bucks, D.A., Erie, L.J., French, O.F., Nakayama, F.S., Pew, W.D., 1980. Subsurface trickle irrigation management with multiple cropping. Trans. Amer. Soc. Agr. Eng. 24(2):14821489.

Coelho, E.F., Or, D. 1999. Root distribution and water uptake patterns of corn under surface and subsurface drip irrigation. Plant Soil 206:123-136.

Fernández, M.D., Thompson, R., Bonachela, S., Gallardo, M., Granados, M.R., 2012. Uso del agua de riego en los cultivos en invernadero. Cuaderno de Estudios Agroalimentarios, Julio 2012: 115-138.

Fernández, M.D., Bonachela, S., Orgaz, F., Thompson, R., López, J.C., Granados, M.R., Gallardo, M., Fereres, E., 2010. Measurement and estimation of plastic greenhouse reference evapotranspiration in a Mediterranean climate. Irrigation Science 28:497509.

Freeman, I., Blackwell, J., Garzoli, K.V., 1976. Irrigation frequency and total water applications with trickle and furrow systems. Agricultural Water Management. 1:21-31.

Gavilán, P., 2014. Evaluación de la uniformidad de cintas de riego en condiciones de campo. Elección del tiempo de riego más adecuado. Jornada Técnica "Mejora y uso del agua de riego en el cultivo de la fresa". Instituto de Investigación y Formación Agraria y Pesquera. Consejería de Agricultura, Pesca y Desarrollo Rural. Junta de Andalucía. Sevilla, Noviembre 2014. (Disponible en www.servifapa.es). 
Gavilán, P., Estévez, J., Berengena, J., 2008. Comparison of standardized reference evapotranspiration equations in Southern Spain. Journal of Irrigation and Drainage Engineering 134(1):1-12.

Gavilán, P., Lozano, D., Ruiz, N., 2014a . El riego de la fresa en el entorno de Doñana. Evapotranspiración, coeficientes de cultivo y eficiencia del riego. XXXII Congreso Nacional de Riegos. Madrid, 10 al 12 de junio de 2014.

Gavilán, P., Ruiz, N., Lozano, D., 2014 . Estimación de la evapotranspiración del cultivo de la fresa basada en pronósticos meteorológicos. Validación con datos experimentales. V Jornadas de Agrometeorología. Valencia 13 y 14 de noviembre de 2014.

Hanson, B.R., May, D.M., Schwankl, L.J., 2003. Effect of irrigation frequency on subsurface drip irrigated vegetables. HortTecnology, 13(1):115-120.

Hutmacher, R.B., Vail, S.S., Muthamia, J.G., Mwaja, V., Liu., R.C., 1985. Effect of trickle irrigation frequency and installation depth on tomato growth and water status, p. 798804. In: Drip/Trickle Irrigation in Action, Proc. 3rd Intl. Drip/Trickle Irrigation Congress.

Linnemannstöns, T., Ançay, A., Baroffio, C.A., 2013. Strawberry drip-irrigation in plastic tunnel - effects of irrigation frequency on water use, yield and fruit quality. Proceedings of International Strawberry Congress, 4-6 September 2013, Antwerp, Belgium.

Lozano, D., Ruiz, N., Gavilán, P., 2014. Evaluación en campo de cintas de riego por goteo de diferente caudal en un cultivo de fresa. Agricultura, noviembre 2014:778-781. 